\title{
ACEITAÇÃO SENSORIAL DE REESTRUTURADOS EMPANADOS ELABORADOS COM FILÉ DE PEITO DE GALINHAS MATRIZES DE CORTE E POEDEIRAS COMERCIAIS ${ }^{1}$
}

\author{
Tatiana Pacheco NUNES ${ }^{2 *}$, Marco Antônio TRINDADE ${ }^{3}$, \\ Edwin Moisés Marcos ORTEGA ${ }^{4}$, Carmen Josefina Contreras CASTILLO ${ }^{5}$
}

\begin{abstract}
RESUMO
Em 2005, no Brasil, o alojamento de galinhas poedeiras comerciais e de matrizes de corte somados foi de 109 milhões de cabeças, sendo que um número próximo a este deve ter sido descartado no mesmo período. Este grande número de galinhas descartadas contrasta com a falta de mercado consumidor e o baixo valor comercial alcançado por estas aves. A utilização da carne de galinhas na elaboração de produtos de conveniência poderia agregar valor e ampliar o consumo desta matéria-prima. Diante do exposto, o objetivo deste trabalho foi elaborar produtos empanados tipo nuggets, utilizando carne de galinhas de descarte (matrizes de corte e poedeiras comercias brancas) e avaliar a aceitação pelo consumidor destes produtos, em comparação com nuggets elaborados com carne de frango. Os produtos desenvolvidos foram avaliados por análise sensorial, utilizando testes de aceitação e de intenção de compra em localização central (400 provadores). Não foram encontradas diferenças ( $p>0,05$ ) na aceitação sensorial nem na intenção de compra entre os três diferentes produtos avaliados. Concluiu-se que a elaboração de produtos empanados pode representar uma boa alternativa para a utilização de filés de peito de galinhas, agregando maior valor a estas aves ao final do ciclo de postura.

Palavras-chave: galinhas de descarte, nuggets, avaliação sensorial.
\end{abstract}

\section{SUMMARY}

SENSORY ACCEPTANCE OF NUGGETS PREPARED WITH BROILER BREEDER AND SPENT LAYER HENS BREAST MEAT. In Brazil, the flock size of layer and broiler breeder hens housed in 2005 was 109 million. A similar number of hens might be spent in this period. This great number of spent hens contrasts with the absence of consumer market and the low commercial value reached by these birds. The manufacture of convenience products using spent hens meat could add value and elevate the consumption of this raw material. Thus, the purpose of this study was to develop nuggets made with spent hens (white layers and broiler breeders) meat and evaluate the consumer acceptance of these products in comparison to nuggets elaborated with broiler meat. Sensory evaluation of acceptance and purchase intent was carried out in central location (400 consumers). No difference ( $p>0.05$ ) were detected by consumers in the acceptance and in the purchase intent tests between the three different nuggets evaluated. It was concluded that nuggets production could represent a good choice for the utilization of hens breast meat at the end their commercial laying cycle.

Keywords: spent hens, nuggets, sensory evaluation.

\section{1 - INTRODUÇÃO}

O volume de material biológico e os custos com o trabalho e o transporte, associados ao abate de galinhas de descarte, fazem com que essa criação se torne um dos principais problemas econômicos e ambientais da indústria avícola [12]. Segundo esse autor, o plantel de galinhas poedeiras nos Estados Unidos é da ordem de 320 milhões de cabeças, as quais valem aproximadamente US\$ 2,69 cada.

\footnotetext{
${ }^{1}$ Recebido para publicação em 3/2/2006. Aceito para publicação em 20/10/2006 (001670)

${ }^{2}$ Departamento de Alimentos e Nutrição Experimental,

Faculdade de Ciências Farmacêuticas, Universidade de São Paulo,

Av. Professor Lineu Prestes, 580, Bloco 14, Cidade Universitária,

CEP 05508-900, São Paulo (SP), Brasil,

E-mail:tpnunes@uol.com.br

${ }^{3}$ Departamento de Engenharia de Alimentos,

Faculdade de Zootecnia e Engenharia de Alimentos,

Universidade de São Paulo

${ }^{4}$ Departamento de Ciências Exatas,

Escola Superior de Agricultura "Luiz de Queiroz",

Universidade de São Paulo

${ }^{5}$ Departamento de Agroindústria, Alimentos e Nutrição,

Escola Superior de Agricultura "Luiz de Queiroz",

Universidade de São Paulo

* A quem a correspondência deve ser enviada
}

Ao final do ciclo de produção, o valor por ave é reduzido para US\$ 0,07. Cinqüenta e cinco porcento dessas aves são abatidas anualmente, sendo destinadas, principalmente, para a produção de sopas e caldos concentrados. Com o crescente aumento na produção de frangos, a venda de matrizes de descarte a preços razoáveis tem se tornado mais difícil, afetando os lucros dos produtores [10]. A distribuição destas aves para consumo doméstico encontra problemas em função do grande tamanho (de 3 a $4 \mathrm{Kg}$ ), excessiva deposição de gordura e aspecto desagradável da pele, além da carne ser mais dura e menos suculenta [10]. Em 2005, segundo as estatísticas da União Brasileira de Avicultura [17], foram alojadas no Brasil 36,7 milhões de matrizes para corte e 72,5 milhões de poedeiras comerciais (ovos brancos e vermelhos). Considerando que o alojamento é a quantidade de aves que são agregadas ao plantel para substituir aves velhas de descarte, um número próximo a este deve ter sido descartado no mesmo período, implicando nos mesmos problemas citados anteriormente. Portanto, a melhor utilização destas galinhas ao final de ciclo de postura, como, por exemplo, pelo processamento de produtos de maior valor agregado, poderia representar vantagens econômicas para a indústria avícola brasileira. 
O perfil do consumidor foi completamente alterado nessas últimas décadas devido à necessidade de se trabalhar fora, com conseqüente redução no tempo dedicado ao preparo de alimentos. Essas mudanças de hábitos exigiram adaptações da indústria avícola para o desenvolvimento de produtos prontos para serem consumidos e de fácil preparo [3]. No Brasil, o interesse por produtos de aves vem crescendo com o decorrer dos anos. Dentre esses produtos, destacam-se os reestruturados empanados, tipo nuggets, cuja elaboração é dada pela desintegração do músculo por processos mecânicos, seguida pela mistura dos pedaços resultantes, para, posteriormente, serem formatados em porções específicas, proporcionarem menor perda durante o cozimento, melhor aproveitamento dos músculos que seriam subutilizados, além de serem fáceis para aquecer e servir, por serem empanados e pré-fritos. O empanamento prolonga a vida útil dos produtos pelo retardamento da oxidação, além de proteger a carne da desidratação e queima pelo frio durante o congelamento [11].

A elaboração de nuggets utilizando carne de peito de galinhas poderia apresentar problemas com relação à textura, visto que animais mais velhos apresentam a carne notadamente mais dura [14]. Outro problema poderia ser causado devido ao aumento da intensidade do aroma apresentado por animais mais velhos [15]. Alguns pesquisadores [2, 7] estudaram a produção de empanados e reestruturados elaborados com carne de galinhas de descarte, porém poucos trabalhos foram realizados no sentido de compará-los a produtos feitos com carne de frango, que é a matéria-prima normalmente utilizada pela indústria.

O principal objetivo desse estudo foi avaliar a aceitação sensorial de nuggets elaborados com carne de peito de galinhas (matrizes de corte e poedeiras comerciais brancas) em comparação com nuggets elaborados com carne de peito de frango.

\section{2 - MATERIAL E MÉTODOS}

\section{1 - Matéria-prima}

Os filés de peito de galinhas foram desossados manualmente a partir das carcaças de galinhas matrizes pesadas (aprox. 3,5 Kg/carcaça) e poedeiras comerciais (aprox. $1,2 \mathrm{Kg} /$ carcaça). Utilizou-se em torno de $150 \mathrm{Kg}$ de cada tipo de galinha, correspondendo a aproximadamente 45 carcaças de matrizes e 110 de poedeiras, em cada repetição. Os filés de peito dos frangos foram desossados a partir de cortes de peito com osso, totalizando aproximadamente $30 \mathrm{Kg}$ de matéria-prima em cada repetição. Todas as desossas foram repetidas três vezes. As respectivas peles de cada ave foram separadas durante a desossa manual e utilizadas como matéria-prima na elaboração dos nuggets.

\section{2 - Processamento}

Foram elaborados três tratamentos diferentes, ou seja: um de frango, um de galinha matriz e um terceiro de galinha poedeira. Em cada tratamento, utilizou-se o filé de peito e a pele da respectiva ave. Todos os processamentos foram realizados em triplicata. A formulação padrão está apresentada na Tabela 1. Os filés de peito foram congelados separadamente e moídos em moedor (marca HERMANN, modelo 106, São Paulo, Brasil), em discos de $10 \mathrm{~mm}$, enquanto a pele foi congelada e moída em discos de $2 \mathrm{~mm}$. Após o processo de moagem, a carne foi processada em misturadeira (marca CAF, modelo M-60, Rio Claro, Brasil) juntamente com os demais ingredientes, por um período de $5 \mathrm{~min}$. Em seguida, a massa foi moldada na forma final dos nuggets em formadora (marca HOLLYMATIC, modelo 54, Countryside, USA). Após a moldagem, os produtos seguiram para a linha de empanamento e pré-fritura (marca STEIN, modelo 100, Sandusky, USA), que contou com as seguintes etapas: primeiramente foram passados em pré-dust, marca KERRY PDA66-TC, para serem, em seguida, imersos em batter (KERRY ABB81), dissolvido na proporção 1:1,6 (pó:água). Para cobertura final, utilizou-se CLASSIC CRUMB 4034, também da KERRY. Os nuggets sofreram uma pré-fritura em gordura vegetal (marca CEVAL, tipo CUKIN FRY) a $180{ }^{\circ} \mathrm{C}$ durante aproximadamente um minuto. Após a pré-fritura, os produtos foram embalados, congelados e armazenados a $-18^{\circ} \mathrm{C}$, para posterior avaliação sensorial.

TABELA 1 - Formulação básica utilizada na fabricação dos nuggets.

\begin{tabular}{lcc}
\hline \multicolumn{1}{c}{ Ingredientes } & \% & Peso (g) \\
\hline Peito & 78,00 & 7.800 \\
Pele & 10,00 & 1.000 \\
Água & 8,02 & 802 \\
Sal & 1,10 & 110 \\
Proteína de soja & 1,00 & 100 \\
Tripolifosfato de sódio & 0,35 & 35 \\
Cebola em pó & 0,15 & 15 \\
Alho em pó & 0,10 & 10 \\
Pimenta branca & 0,03 & 3 \\
Açúcar & 0,20 & 20 \\
Lactato de sódio & 0,80 & 80 \\
Eritorbato de sódio (antioxidante) & 0,25 & 25 \\
Total & 100,00 & 10.000 \\
\hline
\end{tabular}

\section{3 - Análises físico-químicas}

Composição centesimal: a metodologia oficial da AOAC [5] foi utilizada para determinação de proteína, gordura, umidade e cinzas.

Determinação de pH: Medido em pHmetro DIGIMED DM-20 com eletrodo de punção diretamente na amostra.

\section{4 - Análise sensorial}

A avaliação sensorial dos nuggets foi realizada por testes afetivos em localização central. Foram aplicados os testes de aceitação do consumidor e de intenção de compra em três locais diferentes (em dois hipermercados e um supermercado) na cidade de Campinas, visando-se obter dados de diferentes classes econômicas. Um total de 400 consumidores foi recrutado nos próprios locais, momentos antes da realização das análises, excluindo-se apenas 
aqueles provadores que apresentassem aversão ao produto avaliado. Os testes sempre foram realizados no período da tarde e início da noite.

A ficha apresentada aos consumidores continha um questionário inicial para a classificação econômica dos produtos. Esta classificação foi baseada no Critério de Classificação Econômica Brasil de acordo com a Associação Nacional de Empresas de Pesquisa - ANEP [1], que teve como objetivo estimar o poder de compra das pessoas e das famílias urbanas. O sistema adotado baseia-se na soma de pontos, em que a posse de cada item presente na primeira parte da ficha implica na aquisição de pontos que, somados ao grau de instrução do chefe da família, indica a que classe econômica pertence o entrevistado.

Com relação aos produtos, solicitou-se a avaliação global (incluindo aroma, sabor, suculência e textura) da aceitação de cada tipo de produto e, também, da intenção de compra deles, independentemente do preço. A escala sensorial do teste de aceitação variou de 7 a 1 , onde: 7 = gostei muitíssimo; 6 = gostei muito; 5 = gostei; 4 = não gostei $/ \mathrm{nem}$ desgostei; 3 = desgostei; 2 = desgostei muito; e 1 = desgostei muitíssimo. No teste de intenção de compra, utilizou-se uma escala variando de 5 a 1 , onde: 5 = certamente compraria; 4 = provavelmente compraria; 3 = talvez comprasse/talvez não; 2 = provavelmente não compraria; e 1 = certamente não compraria. Na ficha sensorial oferecida, ainda constava um espaço no qual cada provador podia expressar, de forma livre, de quais atributos tinham gostado mais (ou menos) nos produtos consumidos.

Os produtos congelados foram fritos por dois minutos em fritadeira elétrica (marca TEDESCO, modelo FE2B elétrico, Caxias do Sul, Brasil), a $180{ }^{\circ} \mathrm{C}$. Imediatamente após a fritura, os três tipos de nuggets (matriz, poedeira e frango) foram dispostos em uma bandeja de poliestireno expandido branca para serem servidos aos consumidores. Cada tipo foi codificado com algarismos de três dígitos aleatórios, diferentes para cada provador. Para a apresentação das amostras nas bandejas, utilizou-se o delineamento experimental de blocos completos balanceados.

\section{5 - Análises estatísticas}

Para a análise e interpretação dos resultados do teste de aceitação, procedeu-se a uma Análise de Variância e, para as comparações múltiplas, trabalhou-se com o Teste de Tukey. Como análise complementar, utilizou-se a técnica multivariada descritiva de Análise de Correspondência [6]. O nível de significância $\mathrm{p} \leq 0,05$ foi adotado em todas as análises. Para a realização das análises, utilizou-se o software SPSS Professional Statistics 12.0.

\section{3 - RESULTADOS E DISCUSSÃO}

\section{1 - Caracterização dos filés de peito}

Os resultados das análises de composição centesimal dos filés de peito das três diferentes aves estão apresentados na
Tabela 2. O teor de umidade encontrado nos filés de peito de frangos foi maior ( $\mathrm{p} \leq 0,05$ ) quando comparado com os filés de peito de galinhas matrizes, como conseqüência da menor porcentagem de gordura total presente no músculo do frango. Não foi observada nenhuma diferença $(p>0,05)$ nos teores de proteína e cinzas. Esses resultados são similares aos obtidos por outros autores [7, 9].

Não foi observada diferença $(p>0,05)$ nos valores de $\mathrm{pH}$ entre os três diferentes tipos de filés de peito analisados. Os valores de $\mathrm{pH}$ obtidos para o filé do peito de frango estão de acordo com os valores encontrados por diversos autores [4, 8, 13, 16].

TABELA 2 - Composição centesimal média, pH e erro padrão dos diferentes filés de peito utilizados na elaboração dos nuggets.

\begin{tabular}{lccccc}
\hline & Proteína & Gordura & Umidade & Cinzas & $\mathbf{p H}$ \\
\hline Poedeiras & $22,6^{\mathrm{a}} \pm 0,6$ & $1,4^{\mathrm{ab}} \pm 0,2$ & $74,5^{\mathrm{ab}} \pm 0,4$ & $1,0^{\mathrm{a}} \pm 0,1$ & $5,8^{\mathrm{a}} \pm 0,1$ \\
Matrizes & $22,7^{\mathrm{a}} \pm 0,7$ & $2,1^{\mathrm{a}} \pm 0,4$ & $73,9^{\mathrm{a}} \pm 0,5$ & $1,0^{\mathrm{a}} \pm 0,1$ & $5,7^{\mathrm{a}} \pm 0,0$ \\
Frangos & $21,5^{\mathrm{a}} \pm 0,4$ & $0,9^{\mathrm{b}} \pm 0,1$ & $75,8^{\mathrm{b}} \pm 0,1$ & $1,1^{\mathrm{a}} \pm 0,1$ & $5,9^{\mathrm{a}} \pm 0,1$ \\
\hline
\end{tabular}

Médias com letras iguais dentro de uma mesma coluna não apresentaram diferença significativa $(p \leq 0,05)$.

\section{2 - Perfil dos consumidores}

Pela Tabela 3 é possível observar, de uma forma geral, o perfil dos consumidores entrevistados.

TABELA 3 - Perfil dos consumidores entrevistados na avaliação sensorial de nuggets.

\begin{tabular}{|c|c|c|c|c|}
\hline \multirow[t]{2}{*}{ Pergunta } & \multirow{2}{*}{$\begin{array}{l}\text { Opções de } \\
\text { resposta }\end{array}$} & \multicolumn{2}{|c|}{ Consumidores } & \multirow{2}{*}{$\begin{array}{c}\text { Renda média } \\
\text { mensal (R\$) }\end{array}$} \\
\hline & & Número & $\%$ & \\
\hline \multirow{4}{*}{$\begin{array}{l}\text { Qual a sua } \\
\text { idade (anos) }\end{array}$} & menos de 20 & 87 & 22 & \\
\hline & de 21 a 40 & 186 & 46 & \\
\hline & de 41 a 60 & 91 & 23 & \\
\hline & acima de 60 & 37 & 9 & \\
\hline \multirow[t]{2}{*}{ Gênero } & Masculino & 158 & 39 & \\
\hline & Feminino & 243 & 61 & \\
\hline \multirow{5}{*}{$\begin{array}{l}\text { Grau de } \\
\text { instrução } \\
\text { do chefe da } \\
\text { família }\end{array}$} & $\begin{array}{l}\text { analfabeto/primário } \\
\text { incompleto }\end{array}$ & 29 & 7 & \\
\hline & $\begin{array}{l}\text { primário completo/ } \\
\text { ginasial incompleto }\end{array}$ & 66 & 16 & \\
\hline & $\begin{array}{l}\text { ginasial completo/ } \\
\text { colegial incompleto }\end{array}$ & 72 & 18 & \\
\hline & $\begin{array}{l}\text { colegial completo/ } \\
\text { superior incompleto }\end{array}$ & 95 & 24 & \\
\hline & superior completo & 139 & 35 & \\
\hline \multirow{7}{*}{$\begin{array}{l}\text { Classe } \\
\text { econômica }\end{array}$} & A1 & 26 & 6 & 7793,00 \\
\hline & $\mathrm{A} 2$ & 75 & 19 & 4648,00 \\
\hline & B1 & 69 & 17 & 2804,00 \\
\hline & B2 & 104 & 26 & 1669,00 \\
\hline & C & 100 & 25 & 927,00 \\
\hline & $\mathrm{D}$ & 24 & 6 & 424,00 \\
\hline & $\mathrm{E}$ & 3 & 1 & 207,00 \\
\hline
\end{tabular}

Pelos questionários distribuídos em relação aos bens de consumo, foi possível classificar cada consumidor em uma 
classe econômica e a sua respectiva renda média mensal, como pode ser visto no final da Tabela 3. É possível observar que $68 \%$ dos consumidores pertenciam às classes $\mathrm{A}$ e B, diferentemente da distribuição encontrada no Brasil, segundo a ANEP [1], em que as classes A e B somaram quase a metade do valor encontrado nesse trabalho, o equivalente a $30 \%$, sendo este dividido em 1, 5, 8 e 14\% para as classes A1, A2, B1 e B2, respectivamente.

Nota-se que a maior parte pertencia à faixa etária dos 21 aos 40 anos e mais da metade dos consumidores entrevistados pertencia ao sexo feminino. Agrupando as respostas por sexo e faixa etária, conforme apresentado na Tabela 4 , observa-se que a grande diferença entre homens e mulheres ocorreu na faixa etária em que se situavam as pessoas economicamente ativas, ou seja, entre os 21 e 60 anos. Esses consumidores significaram $69,5 \%$ do total entrevistado, sendo $44,6 \%$ do sexo feminino. Deste modo, intensifica-se a idéia de que as mulheres, apesar de trabalharem fora e dedicarem menos tempo no preparo das refeições, ainda são as grandes responsáveis pelo abastecimento alimentar da família, justificando, desse modo, o aumento do consumo dos produtos de conveniência nos últimos anos.

Observa-se também que, apesar da grande maioria dos consumidores entrevistados pertencerem ao sexo feminino, essa diferença acaba sendo pequena, quando analisada isoladamente a porcentagem de consumidores situada nas faixas etárias, menor que 20 e maior que 60 anos.

TABELA 4 - Sexo e faixa etária dos consumidores.

\begin{tabular}{lccccc}
\hline \multirow{2}{*}{ Faixa etária } & \multicolumn{2}{c}{ Homens } & & \multicolumn{2}{c}{ Mulheres } \\
\cline { 2 - 3 } \cline { 5 - 6 } & número & $\%$ & & Número & $\%$ \\
\hline Menor que 20 anos & 47 & 12 & & 40 & 10 \\
$21-40$ anos & 70 & 17 & & 116 & 29 \\
$41-60$ anos & 24 & 6 & & 67 & 17 \\
Maior que 60 anos & 17 & 4 & & 20 & 5 \\
Total & 158 & 39 & & 243 & 61 \\
\hline
\end{tabular}

A análise da freqüência de consumo em relação à classe econômica, observado na Figura 1, indicou um agrupamento das classes em função do seu poder de compra, ou seja, as classes de maior poder aquisitivo tiveram suas respostas relacionadas a um consumo mais freqüente de nuggets quando comparadas com as classes com menor poder de compra.

Também foi observado que, independente da freqüência de consumo, as classes A e B representaram um consumo superior a $70 \%$ do total da população entrevistada. Tais resultados demonstram que os nuggets, apesar de serem produtos de grande aceitação, ainda são considerados relativamente caros para a população de países em desenvolvimento, implicando a necessidade da pesquisa de novos produtos com matérias-primas mais baratas. Quando se analisou a freqüência de consumo independente das classes econômicas, observou-se que a maioria dos consumidores entrevistados (31\%) consumia nuggets até uma vez por se-

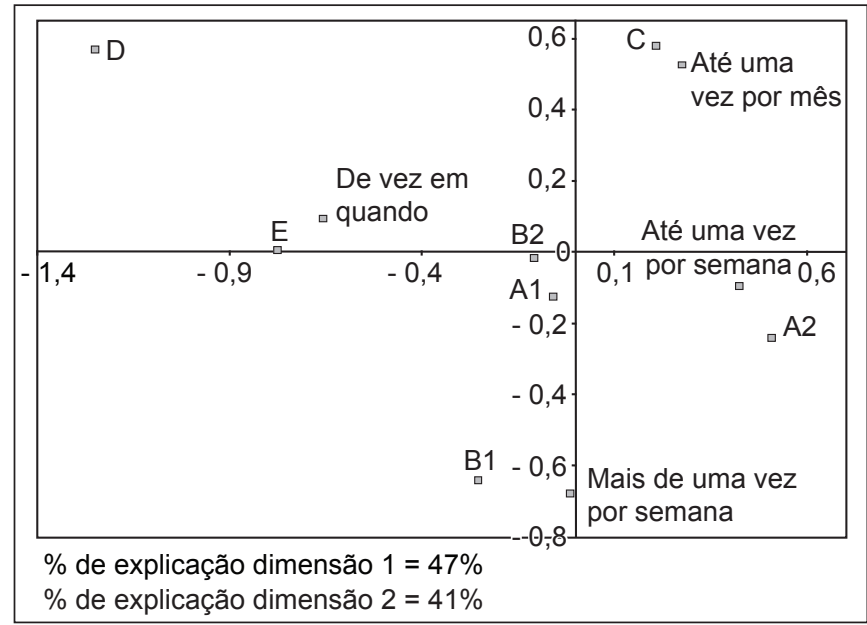

FIGURA 1 - Análise de correspondência entre a freqüência de consumo e a classe econômica dos provadores.

mana (Tabela 5). Deste modo, também é possível observar que, apesar de ainda serem considerados caros pela maioria dos entrevistados, há uma popularização desses produtos em função da sua importância na dieta alimentar, à medida que trazem praticidade para o dia-a-dia.

TABELA 5 - Freqüência de consumo de produto tipo nuggets citada pelos provadores

\begin{tabular}{lcc}
\hline \multicolumn{1}{c}{ Freqüência de consumo } & Número de respostas & $\%$ \\
\hline Mais de uma vez por semana & 76 & 19 \\
Até uma vez por semana & 152 & 31 \\
Até uma vez por mês & 91 & 23 \\
De vez em quando & 107 & 27 \\
\hline
\end{tabular}

\section{3 - Avaliação sensorial afetiva dos produtos}

A avaliação sensorial dos nuggets, considerando todos os provadores, indicou que não houve diferença ( $p>0,05)$ na aceitabilidade entre os três tratamentos (frango, matriz e poedeira) avaliados. Em função da grande porcentagem de consumidores pertencentes a faixa etária de 21 a 40 anos (46\% do total), também se realizou a ANOVA entre as amostras por faixas etárias, pois uma possível diferença neste sentido poderia ser importante quando se pensa em estratégias de marketing para um determinado público alvo. No entanto, também não foram encontradas diferenças ( $p>0,05$ ) entre os produtos analisando-se separadamente todas as faixas etárias (até 20 anos, de 21 a 40 anos, de 41 a 60 anos e maiores que 60 anos). Como a carne de galinha possui uma textura mais dura, menos suculenta e um sabor intenso, imaginava-se, inicialmente, que os consumidores pudessem detectar diferença entre as amostras analisadas. As médias de aceitação para frango, poedeira e matriz foram, respectivamente, 6,$4 ; 6,3$; e 6,3 . Isto significa que, além de não terem sido detectadas diferenças na aceitabilidade entre as amostras, todas foram muito bem aceitas entre os provadores, ficando estes valores na escala entre o "gostei muito" (6) e o "gostei muitíssimo" (7). 
Na Figura 2, está apresentada a distribuição das notas atribuídas pelos consumidores e, como pode ser observado, os três tipos de nuggets foram avaliados, por mais de $90 \%$ dos consumidores entrevistados, com classificação entre o "gostei" e "gostei muitíssimo".

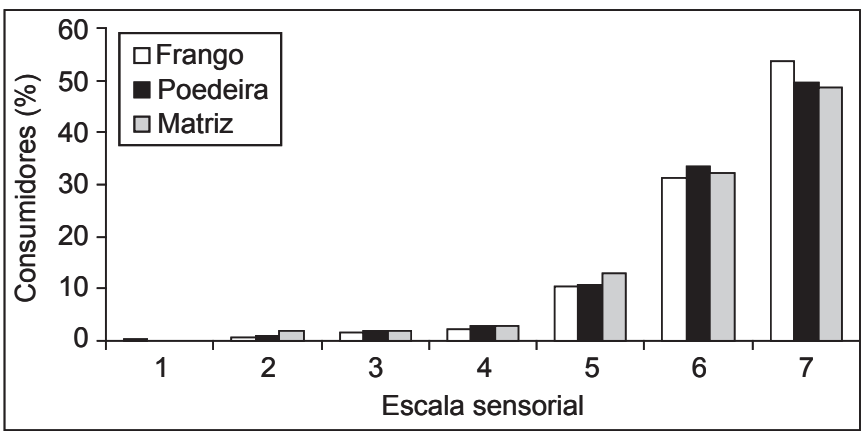

FIGURA 2 - Distribuição da porcentagem de consumidores em relação ao índice de aceitação dos nuggets ( 1 - desgostei muitíssimo e 7-gostei muitíssimo)

Em relação ao espaço reservado aos consumidores para que pudessem expressar livremente os atributos que mais os agradassem durante a análise, foi observado que, das 365 respostas obtidas espontaneamente, $61,6 \%$ dos consumidores escreveram sabor, seguido da suculência, crocância e maciez com 10,1, 7,7 e 6,6\%, respectivamente, como os preferidos. Os atributos como textura, dureza da carne, odor, tempero e seco foram lembrados nessa ordem com 3,$8 ; 3,6 ; 3 ; 2,5$; e $1,1 \%$, respectivamente. Todos esses atributos foram escolhidos independente da matéria-prima utilizada.

Também foi observado que, na tentativa de detectar diferença entre os produtos, muitos consumidores fizeram comentários de forma aleatória em relação às diferenças no tempero e no teor de sal entre os nuggets elaborados com carne de frango, poedeira e matriz. No entanto, os três tipos de nuggets foram elaborados utilizando-se a mesma formulação em termos percentuais de matérias-primas, ingredientes e aditivos, incluindo o teor de sal e de condimentos. Além disso, apesar da composição centesimal dos filés (Tabela 2) terem apresentado diferenças significativas, os percentuais de proteína, gordura, umidade e cinzas para os três tipos de matérias-primas utilizadas foram muito próximos. Ou seja, como não havia uma diferença relevante em termos de composição para as três formulações, pode-se concluir que estes comentários não refletem uma diferença específica entre os atributos sensoriais dos produtos analisados e, conseqüentemente, a não preferência de um produto em relação ao outro. Os dados obtidos estão de acordo com trabalho realizado por ROUSELLE et al. [15]. Estes pesquisadores não encontraram diferenças significativas entre nuggets feitos com carne de peito de frango e de galinhas poedeiras aplicando o teste de consumidor.

Em relação à intenção de compra, também não foi observada diferença significativa $(\mathrm{p} \geq 0,05)$ entre os três produtos avaliados (frango, galinha poedeira e galinha matriz), tanto quando se considerou todos os provadores (400), como quando se comparou as amostras por divisão de faixa etária. A nota média obtida por todos os produtos foi igual, o que os posiciona na escala entre os critérios “certamente compraria" (5) e "provavelmente compraria" (4). Deste modo, pode-se notar a mesma tendência observada na análise de aceitação sensorial, em que os diferentes tipos de nuggets analisados obtiveram não apenas notas muito semelhantes entre si, como também ficaram próximos da nota máxima.

Diferentemente do que foi encontrado para a aceitabilidade dos produtos, o teste de análise de variância indicou que a intenção de compra não foi influenciada significativamente $(\mathrm{p} \geq 0,05)$ pela freqüência de consumo, sexo, faixa etária ou classe econômica dos provadores para nenhum dos três tipos de nuggets analisados.

Na Figura 3, observa-se que mais de 50\% dos entrevistados certamente comprariam os produtos, intensificando, deste modo, a idéia de que não foi detectada diferença entre os produtos, já que a distribuição dos consumidores entre os critérios de avaliação é muito próxima entre si, para os três tipos de nuggets.

No presente trabalho, os valores de aceitabilidade mostraram correlação significativa $(\mathrm{p} \leq 0,05)$ com a intenção de compra, para os produtos elaborados com a mesma matériaprima, para a carne de frango a correlação foi de 0,64 e para os de carne de poedeira e matriz foram, respectivamente, 0,58 e 0,67 . Isso significou que os produtos bem aceitos pelos consumidores seriam certamente comprados no mercado.

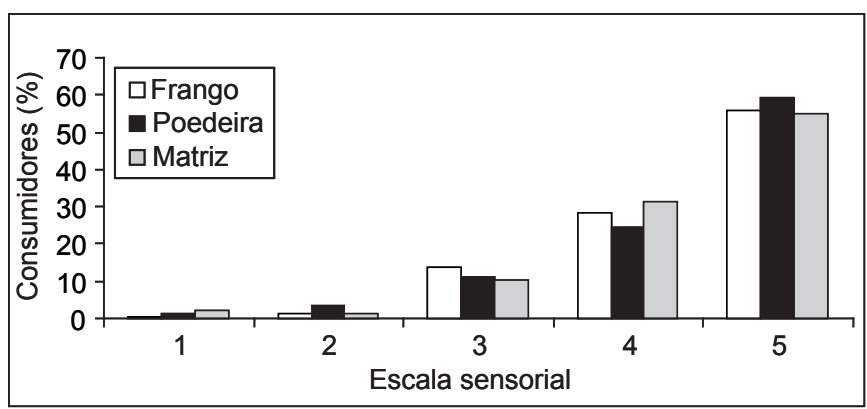

FIGURA 3 - Distribuição da porcentagem dos consumidores em relação à intenção de compra dos nuggets. (1- certamente não compraria e 5- certamente compraria)

\section{4 - CONCLUSÕES}

A elaboração de nuggets com carne de peito de galinha de descarte, tanto de matrizes de corte quanto de poedeiras comerciais, resultou em produtos com qualidade sensorial tão boa quanto a dos nuggets elaborados com filé de peito de frango. Assim, a elaboração de produtos reestruturados empanados pode representar uma boa alternativa para a utilização de filés de peito de galinhas de descarte, agregando maior valor a estas aves ao final do ciclo de postura.

\section{5 - REFERÊNCIAS BIBLIOGRÁFICAS}

[1] ASSOCIAÇÃO BRASILEIRA DE EMPRESAS DE PESQUISA. CCEB - Critério de Classificação Econômica 
Brasil. Disponível em:<http://www.abep.org.br $>$. Acesso em: 09 maio 2001.

[2] BAKER, R. C.; O’BRIEN, S. W.; GOSSETT, P. W. Development and evaluation of chicken burger formulations and effect of beating time incorporating underutilized poultry meat. Poultry Science, v. 63, n. 5, p. 938-948, 1984.

[3] BARBUT, S. Poultry products: formulations and gelation. In: BARBUT (Ed.) Poultry products processing: an industry guide. Boca Raton: CRC Press, 2002. p. 249-289.

[4] BERAQUET, N. J. Carne mecanicamente separada de aves. In: SEMINÁRIO E CURSO TEÓRICO-PRÁTICO - AGREGANDO VALOR À CARNE DE AVES, 1, 2000, Campinas. Anais... Campinas: ITAL, 2000. p. 23-24.

[5] CUNNIF, P. (Ed.). Official methods of analysis of AOAC International. 16. ed. Gaithersburg: AOAC International, 1998. v. 1, p. 26-27.

[6] GREENACRE, M. J. Theory and applications of correspondence analysis. 2. ed. London: Academic Press, 1984. $288 \mathrm{p}$.

[7] HOLLENDER, R.; MacNEIL, J. H.; MAST, M. G. Effect of fragmentation and formulation on the quality of patties made from restructured spent layer meat. Journal of Food Science, v. 52, n. 2, p. 290-293, 1987.

[8] JONES, J. M.; GREY, T. C. Influence of processing on product quality and yield. In: MEAD, G.C. (Ed.). Processing of poultry. London: Chapman \& Hall, 1995. p. 127-130.

[9] KONDAIAH, N.; PANDA, B. Processing and utilization of spent hens. World's Poultry Science Journal, v.48, n. 2, p. 255-268, 1992.

[10] KONDAIAH, N. Products from spent hen. Poultry International, v. 49, n. 1, p. 46-47, Aug. 1993.

[11] LEMOS, A. L. S. C. Valor agregado e conveniência para produtos cárneos. In: SEMINÁRIO E CURSO TEÓRI-
CO-PRÁTICO AGREGANDO VALOR A CARNE DE AVES, 1, 2000, Campinas. Anais... Campinas; ITAL, 2000. p. 17-19.

[12] LYONS, J. J. Spent hen utilization. e-DIGEST, St. Paul, v.1, n.7, 2001. Disponível em: <http://www.wattnet. com/library/download/eD7sphened.pdf.> Acesso em: 18 março 2005.

[13] QIAO, M.; FLETCHER, D. L.; SMITH, D. P.; NORTHCUTT, J. K. The effect of broiler breast meat color on $\mathrm{pH}$, moisture, water hold capacity and emulsification capacity. Poultry Science, v. 80, n. 6, p. 676-680, 2001.

[14] ROLAND, L. M.; SEIDEMAN, S. C.; DONNELLY, L. S.; QUENZER, N. M. Physical and sensory properties of chicken patties made with varying proportions of white and dark spent fowl meat. Journal of Food Science, v. 46, n. 3, p. 834-837, 1981.

[15] ROUSELlE, J. R.; SEACAT, K.; KIEME, A. I.; STADELMAN, W. J. Utilizing flake-cut spent fowl meat in chicken patties. Poultry Science, v. 63, n. 5, p. 932-937, 1984.

[16] SAMS, A. R.; MILLS, K. A. The effect of feed withdrawal duration on the responsiveness of broiler pectoralis to rigor mortis acceleration. Poultry Science, v. 72, n. 10, p.1789-1796, 1993.

[17] UNIÃO BRASILEIRA DE AVICULTURA. Últimos números da avicultura. Disponível em: $<$ http://www.uba.org. br $>$. Acesso em: 19 janeiro 2006.

\section{6 - AGRADECIMENTO}

À Fundação de Amparo à Pesquisa do Estado de São Paulo (FAPESP) por viabilizar a execução do Projeto: Características físico-químicas, microbiológicas, nutricionais e tecnológicas de carne mecanicamente separada de galinhas de descarte (Projeto $n^{\circ}$ 99/11489-1). 